\title{
СТРАТЕГИЧЕСКИЙ АНАЛИЗ БИЗНЕС-СРЕДЫ ПРЕДПРИЯТИЯ С ИСПОЛЬЗОВАНИЕМ СОВРЕМЕННЫХ АНАЛИТИЧЕСКИХ ИНСТРУМЕНТОВ
}

\author{
Щеголеватых Наталья Леонидовна \\ Аспирант, Российский экономический университет \\ имени Г.В. Плеханова \\ tomexspert@mail.ru
}

\section{STRATEGIC ANALYSIS OF THE BUSINESS ENVIRONMENT OF THE ENTERPRISE USING MODERN ANALYTICAL TOOLS}

\section{N. Shchegolevatykh}

Summary. The article is devoted to the study of the features of conducting a strategic analysis of the enterprise's business environment using modern analytical tools. An indicative list of applied problems of researching the business environment of the enterprise and the corresponding analytical approaches have been highlighted. As a modern analytical toolkit, data mining is considered, and the algorithm for its use is also formalized. On the example of assessing the external environment of an enterprise selling cereal products, applied aspects of using data mining are indicated.

Keywords: business environment, enterprise, data mining. и зменчивость и неопределенность функционирования современных хозяйственных образований, непрерывное возникновение новых тенденций в развитии экономики и общества, ускорение научно-технического прогресса создают принципиально новую бизнес-среду, которая значительно отличается от той, в которой происходило формирование и развитие предприятий до недавнего времени [1].

В таких условиях все большую актуальность приобретает проблема повышения эффективности стратегического управления, которое, в свою очередь, невозможно правильно осуществлять без проведения соответствующего стратегического анализа бизнес-среды. Именно по результатам стратегического анализа окружения предприятия определяются его потенциальные возможности для развития, обосновывается система стратегий, направленных на усиление позиций в рыночной среде, происходит выбор и обоснование инструментов, позволяющих уменьшить и преодолеть имеющиеся и потенциальные угрозы [2].

С учетом вышеизложенного, не подлежит сомнению тот факт, что стратегический анализ внешней и внутренней среды должен быть многофакторным и системным.
В тоже время, следует отметить, что разнообразие условий и требований к результатам анализа обусловливают необходимость использования широкого спектра соответствующих методов. Однако на современном этапе очень часто возникают нестандартные, неформализованные ситуации, характерные для кризисных явлений в экономике, исследование и оценка которых с использованием традиционных статистических и экономико-математических методов и моделей является слишком сложным и недостаточно результативным процессом.

Поэтому с целью проведения более глубокого и качественного анализа, оценки и прогнозирования бизнес-среды предприятия представляется целесообразным использовать новые аналитические инструменты и подходы обработки экономической информации.

В процессе комплексного анализа бизнес-среды предприятия с использованием современных аналитических инструментов оценивается текущее состояние внутренней и внешней среды его функционирования, а также параллельно проводится причинно-следственный анализ изменения основных показателей под действием факторов влияния [3]. Однако на практике воз- 
Таблица 1. Ориентировочный перечень прикладных задач исследования бизнес-среды предприятия и соответствующие им аналитические подходы

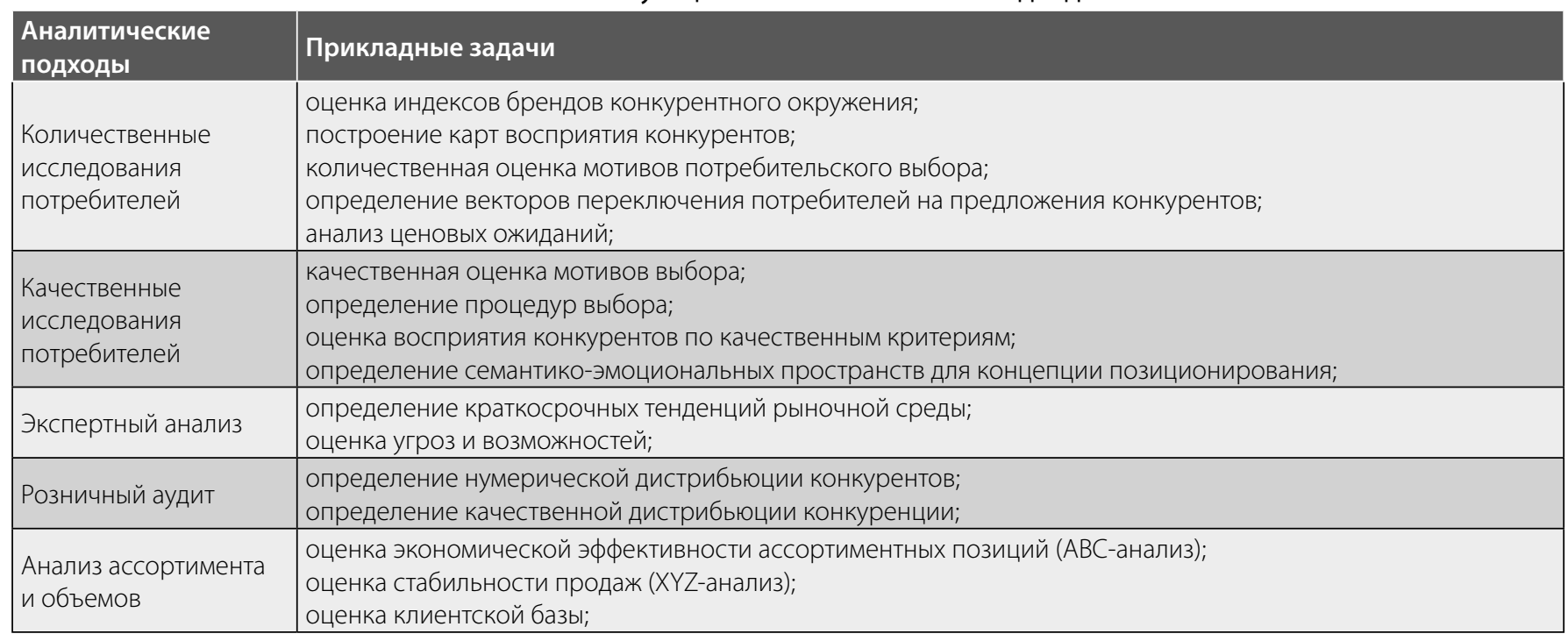

никает ряд вопросов при использовании современных методов анализа, что и обуславливает выбор темы данной статьи.

Вопросы аналитического обоснования решений в зависимости от изменений во внешней и внутренней среде предприятий нашли отражение во многих научных разработках таких отечественных и зарубежных ученых, как: П.Л. Гордиенко, И. Ансофф, Г.Л. Кармин, Н.И. Костина, Л.А. Костырко и другие. Методики стратегического анализа, используемые в деятельности предприятий, широко освещены в работах Голоскокова А., Бабича Т., Бабенко И., Круглова В.В., Матвийчука А. В., Божича В.И.

Однако, отдавая должное важности и значимости наработок, в которых раскрывается теоретико-методологическая база стратегического анализа бизнес-среды предприятий, а также достижения, касающиеся внедрения в практику их деятельности передовых аналитических процедур и подходов, необходимо отметить, что до сих пор существуют вопросы относительно алгоритмов и порядка их использования, которые не получили достаточного освещения и требуют более подробного рассмотрения.

Таким образом, с учетом вышеизложенного, цель статьи заключается в исследовании особенностей проведения стратегического анализа бизнес-среды предприятия с использованием современных аналитических инструментов.

Для принятия управленческих решений на уровне выработки стратегии конкурентного поведения предприятия, разработки программы охвата рынка, пози- ционирования, выбора перспективных направлений функционирования следует провести ряд прикладных исследований окружающей среды. При этом результаты традиционного, фундаментального анализа являются первичными по отношению к прикладным исследованиям с точки зрения целей и задач. Другими словами, в результате фундаментального исследования формируется перечень целей и задач, которые необходимо решить на уровне прикладных исследований с использованием современного аналитического инструментария.

В табл. 1 приведен возможный перечень аналитических подходов и типовые задачи, которые могут быть решены с их помощью.

Традиционные методы анализа бизнес-среды, такие как, функциональная блок-схема потока, схема контроля, Диаграмма Ганта, PERT-диаграммы, PEST-анализ, (STEP-анализ), TEMPLES, матрица «вероятность усиления фактора - воздействие фактора на предприятие» появились еще в XX столетии [4]. Однако в отечественной и зарубежной экономической литературе на сегодняшний день не выявлено единого подхода к оценке влияния бизнес-среды на стратегическую деятельность предприятия. Среди экономистов также отсутствует единое мнение относительно степени влияния каждой группы факторов на стратегию предприятия.

В настоящее время особую популярность у аналитиков и экспертов в качестве современного аналитического инструментария приобретает интеллектуальный анализ данных (DM, data mining), который является составной частью процесса извлечения знаний из баз данных (KDD, knowledge discovery in data bases) [5]. Он 
Таблица 2. Факторы бизнес-среды предприятия и коэффициенты их значимости

\begin{tabular}{ll} 
Факторы бизнес-среды & Коэффициент значимости (К1) \\
\hline Экономические факторы & 0,35 \\
\hline Политические факторы & 0,30 \\
\hline Технологические факторы & 0,20 \\
\hline Социально-демографические факторы & 0,05 \\
\hline Экологические факторы & 0,05 \\
\hline Культурные факторы & 0,05 \\
\hline Всего & 1,0
\end{tabular}

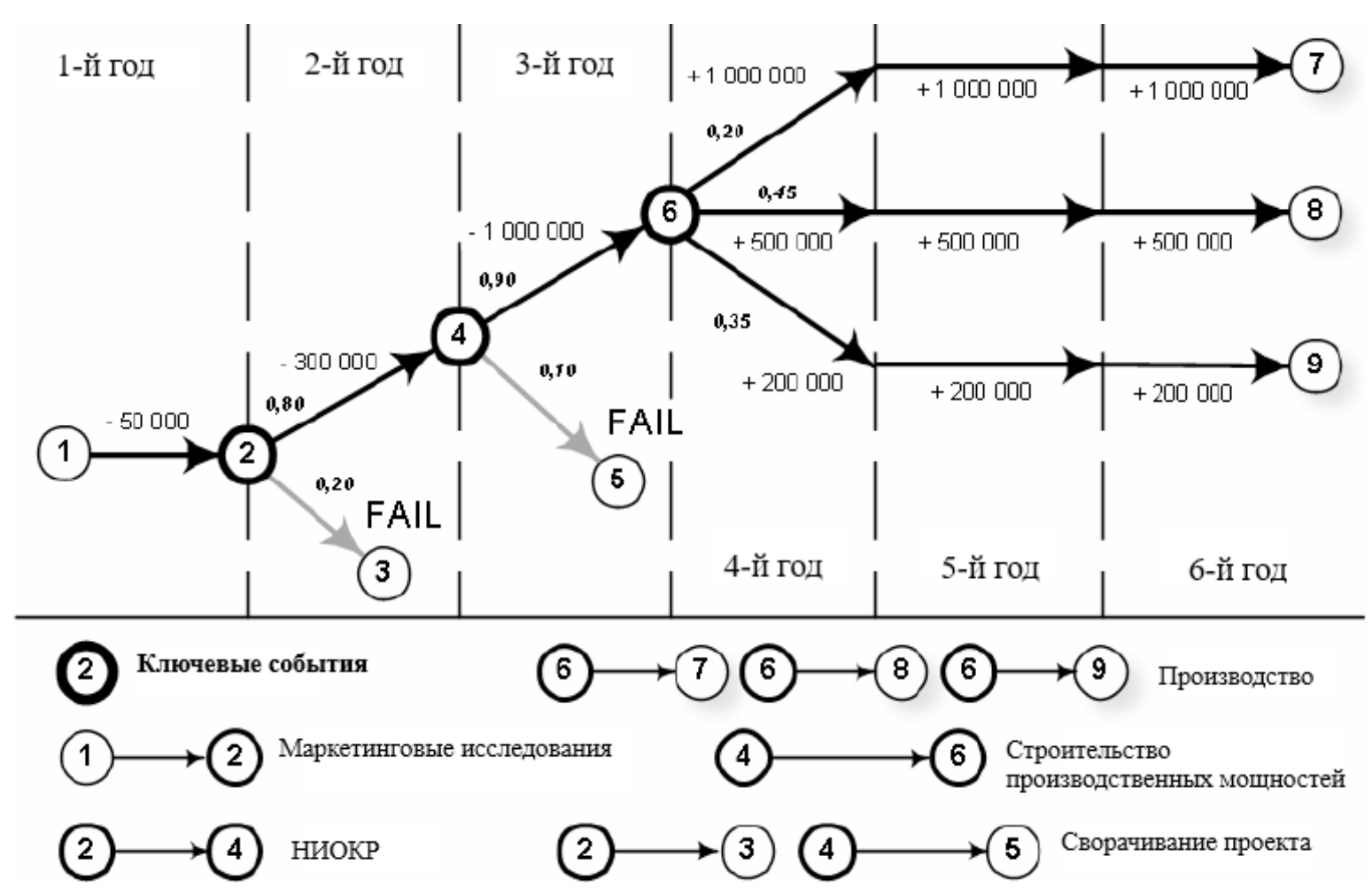

Рис. 1. Модель анализа внешней бизнес-среды предприятия для выхода на новый рынок

позволяет раскрыть суть скрытых зависимостей в данных, выявить взаимные влияния между свойствами внутренней и внешней среды субъектов хозяйствования, информация о которых хранится в базах данных, выделить закономерности, присущие определенному набору данных и ситуации на рынке.

На сегодняшний день уже есть определенные наработки и алгоритмы, позволяющие на практике использовать интеллектуальный анализ данных в процессе оценки и исследования бизнес-среды предприятия. По- следовательность использования данного аналитического инструментария включает в себя следующие этапы:

1. Генерирование альтернативных сценариев функционирования бизнес-среды предприятия с помощью матрицы стратегических альтернатив. Для подготовки сценариев используются предварительно собранные аналитические материалы. Создание сценария предполагает: определение временного интервала хода событий и факторов влияния, словесное толкование их сущности, проведение количественной оценки [6]. 
2. Выбор типа сценариев и критерия отбора факторов моделирования поведения бизнес-среды предприятия. Обычно для сканирования бизнес-среды предприятия выделяют шесть основных сегментов среды, влияние которых необходимо отслеживать, прогнозировать и оценивать на разных структурных уровнях экономики (табл. 2): экономические факторы, политические факторы, технологические факторы, социально-демографические факторы, экологические факторы, культурные факторы.

Безусловно, влияние каждой группы факторов на бизнес-среду и соответственно стратегию предприятия будет разным. В табл. 2. представлены корреляционные коэффициенты значимости группы факторов $\left(K_{l}\right)$.

На основании данных таблица 2, проводится отбор факторов, событий и показателей по всему их разнообразию на каждом структурном уровне экономики, которые прямо или косвенно влияют на предприятие. Анализ каждой группы факторов позволяет сформировать перечень детерминант, которые могут положительно или отрицательно сказаться на развитии предприятия [7].

3. Составление словесного описания сущности каждой стратегической альтернативы и их SWOT-aнализ.

4. Проведение маркетингового исследования ожиданий и преференций целевых потребителей. Речь идет о «полевых исследованиях», которые осуществляются с целью сбора дополнительной первичной информации, обработка которой даст основу для принятия решение на следующем этапе.

5. Экономическое оценивание, составление дерева целей и выбор приемлемой бизнес-модели исследуемого предприятия.

В качестве примера использования современных аналитических инструментов для анализа бизнес-среды предприятия можно привести оценку риска внешнего окружения субъекта хозяйствования в процессе выхода с новым товаром на зарубежный рынок.

Итак, в процессе предварительного анализа бизнес-среды предприятие установило факт наличия неудовлетворенного спроса на золошлаковые материалы на рынке Беларуси (см. рис. 1).

Установлено, что необходимо введение в действие новых производственных мощностей, которые будут производить продукт, отвечающий рыночным стандартам Беларуси необходимо 6 лет. Гипотетически процесс создания и запуска такой линии разделим на четыре ключевых этапа. Рассмотрим более подробно описание этих этапов.

В рамках первого этапа проводится анализ условий ведений бизнеса с целью определения размера потенциального спроса на продукт, также оцениваются существующие на рынке конкуренты, их особенности и возможности. Завершением данного этапа является расчет емкости рынка продукта. Расходы на подобные исследования составляют 50000 долл. США.

На втором этапе вырабатываются варианты развития проекта: 1) завершение проекта (вероятность 0,2), в тому, случае, если в процессе исследования было выявлено отсутствие спроса на продукт или 2) начало проведения исследовательских работ (вероятность 0,8, стоимость 300000 долл. США) если были установлено перспективы реализации данного продукта на рынке.

Третий этап предполагает два возможных пути дальнейшего развития проекта:

1. завершение проекта (вероятность 0,1 ) если результаты прогнозирования выявили невозможность запуска производственной линии по каким-либо причинам;

2. внедрение производственных мощностей для выпуска золошлакогового материала заданного качества (вероятность 0,9).

На четвертом этапе предполагается, что проект начнет генерировать поступление денежных ресурсов. Размер поступлений зависит от ситуации на рынке круп Беларуси. Предположим, эксперты составили три прогноза развития ситуации на рынке:

1. оптимистический (вероятность 0,20, поступления в год \$1,0 млн. долл.),

2. нормальный (вероятность 0,45 , поступления в год \$0,5 млн. долл.),

3. пессимистический (вероятность 0,35, поступления в год \$0,2 млн. долл.).

В результате моделирования было установлено, что на рыночную ситуацию будут влиять обозначенные входные потоки - внутреннее производство в стране, куда будет направляться экспорт? и внутренний спрос в стране.

Таким образом, использование интеллектуального анализа данных для исследования бизнес-среды предприятий позволяет получать более точные прогнозы по сравнению с другими методами, поскольку они дают возможность учитывать качественные изменения, а также определять проблемные стороны деятельности предприятия, требующие усовершенствования или реформирования. 


\section{ЛИТЕРАТУРА}

1. Синявин В.Ю., Васин С. М., Скворцова В. А., Тактарова С. В. Факторный анализ в исследовании деловой активности и развития бизнес-среды в сфере промышленности России // Известия высших учебных заведений. Поволжский регион. Общественные науки. 2019. № 3(51). С. 161-169.

2. Cai, Xin Exploration on the financing risks of enterprise supply chain using Back Propagation neural network // Journal of computational and applied mathematics. 2020. Volume 367: March 15; pp. 13-23.

3. Арипов 0. А. Структурные элементы деловой среды и их влияния на функционирования субъектов малого бизнеса и предпринимательства // Региональные проблемы преобразования экономики. 2019. № 8 (497). С. 184-191.

4. Белевцев А.М., Дворецкий В. В. Методы мониторинга конкурентного окружения высокотехнологичного предприятия // Наукоемкие технологии. 2019. T. 20. № 3. C. 17-23.

5. Шилинскайте И. А. Разработка стратегических направлений развития организации // Известия Международной академии аграрного образования. 2019. № 47. C. 105-106.

6. Устаев Р.М., Чумаченко Р. Г. 06 анализе методик оценки факторов внешней среды и степени ее влияния на организацию // Теория и практика современной науки. 2019. № 5(47). С. 587-593

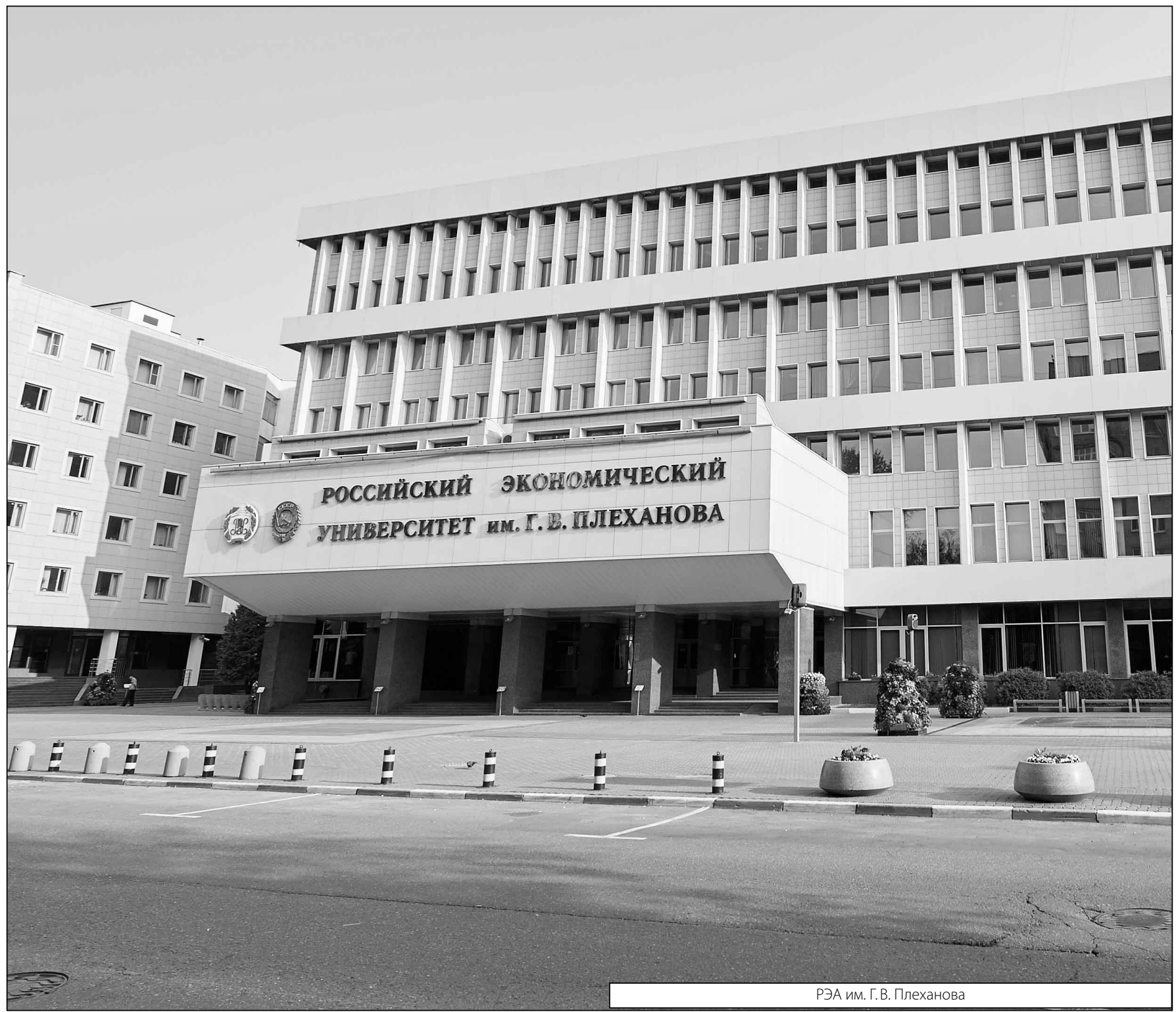

